ÉGYPTE monde arabe

\section{Égypte/Monde arabe}

$21 \mid 1995$

Économie égyptienne et perspectives de paix au

Proche-Orient

\title{
Les flux économiques de la paix et leur impact sur les connexions régionales de l'Égypte
}

Jacques Seguin

\section{OpenEdition}

\section{Journals}

Édition électronique

URL : https://journals.openedition.org/ema/374

DOI : $10.4000 /$ ema.374

ISSN : 2090-7273

Éditeur

CEDEJ - Centre d'études et de documentation économiques juridiques et sociales

Édition imprimée

Date de publication : 31 mars 1995

Pagination : $31-48$

ISSN : 1110-5097

\section{Référence électronique}

Jacques Seguin, « Les flux économiques de la paix et leur impact sur les connexions régionales de l'Égypte », Égypte/Monde arabe [En ligne], 21 | 1995, mis en ligne le 08 juillet 2008, consulté le 07 juillet 2022. URL : http://journals.openedition.org/ema/374 ; DOI : https://doi.org/10.4000/ema.374

Ce document a été généré automatiquement le 7 juillet 2022

Tous droits réservés 


\title{
Les flux économiques de la paix et leur impact sur les connexions régionales de l'Égypte
}

\author{
Jacques Seguin
}

1 Le système régional du Proche-Orient, défini par les champs de force des relations internationales, est en proie à de profondes mutations géopolitiques: des avancées significatives dans le règlement d'un conflit vieux d'un demi-siècle vont faire l'objet de redistributions territoriales et modifier la carte politique de la région syropalestinienne. Les répercussions de cette nouvelle configuration, en particulier la fin de l'état de belligérance et l'intégration politique de deux entités étatiques, l'État d'Israël et l'État palestinien, devraient en affecter l'organisation économique. L'Égypte occupe dans cet environnement régional une place particulière, de par son poids démographique d'abord, de par son influence politique ensuite. Aussi faut-il se demander quels peuvent être les effets de ce nouvel équilibre géopolitique sur son économie.

2 La réponse à cette question dépasse en réalité le cadre de cette étude, et l'analyse que nous proposons ne prétend pas à l'exhaustivité. L'on cherchera plutôt à éclairer, par une approche synthétique, les articulations économiques du système régional. Pour reprendre les propositions de la démarche systémique en géographie, il s'agira de réfléchir aux conséquences de l'intégration d'Israël et de la Palestine en analysant l'économie régionale à travers ses interactions, ses liaisons, les flux qui organisent les réseaux, plutôt que les structures en tant que telles. C'est en termes de feed-back, de rétroaction, que l'impact de la modification du système économique régional sur l'Égypte sera pris en compte. L'on examinera, en particulier, quelles sont les connexions économiques et géoéconomiques de ce pays qui sont susceptibles d'être affectées par la nouvelle configuration d'un ensemble régional plus ouvert aux échanges. Certes, les frontières ne disparaîtront pas, mais leur attribut militaire perdra son caractère exclusif au profit des fonctions économiques, dans un processus de normalisation. Les conséquences de la mutation en cours, analysées par le biais des 
connexions et des flux, seront donc ici essentiellement de nature géoéconomique. Plus ou moins matérialisées, inscrites dans l'espace, les relations économiques sont aussi le vecteur de transformations spatiales à l'échelle d'un pays ou d'une région. C'est sur le système spatial égyptien lui-même que les répercussions peuvent être aussi décelées dans le cadre des nouvelles articulations économiques.

3 Le système économique régional peut être défini comme un ensemble d'acteurs économiques réifiés ou non dans l'État, qui agissent dans des réseaux d'échange de différentes natures. Les interrelations ont trait à diverses variables macro-économiques comme les échanges commerciaux, de services, les mouvements de capitaux publics ou privés, ainsi qu'à des flux de matières premières et énergétiques. L'ensemble des réseaux plus ou moins spatialisés ordonnent la structure et la font évoluer.

4 L'intégration dont il est question signifie l'apport d'éléments nouveaux dans le système économique régional. Les déséquilibres induits vont provoquer des réactions, des rétroactions, c'est-à-dire qu'ils vont susciter un ensemble de décisions individuelles, engageant ou non les collectivités, qui engendreront à leur tour de nouvelles dynamiques spatiales et économiques. Les nouveaux éléments vont se connecter d'une façon ou d'une autre, d'abord entre eux puis avec les structures préalablement existantes. En premier lieu, donc, la structure du système change, mais surtout, son fonctionnement va se trouver altéré par la présence de nouvelles connexions. C'est l'ensemble des réseaux qui peut ainsi en être affecté. Ce qui nous intéresse ici est donc de voir dans quelle mesure l'insertion dans le système régional d'une économie israélienne et, à plus long terme, d'une économie palestinienne va modifier les réseaux de ce système initial et quelles peuvent en être les conséquences sur la place et le rôle de l'Égypte dans l'organisation régionale des flux.

Le système économique régional et ses articulations en termes de flux

5 Pour parler sans ambiguïté, il est nécessaire de mieux cerner ce que l'on entend par «système économique régional ». Le système régional au Moyen-Orient a été défini comme une partie du système mondial, dans un champ d'analyse exploré par les spécialistes des relations internationales. Dans ce cadre, les critères de délimitation font une large place aux rapports de force interétatiques. C'est le degré d'interactions entre les États, à travers les conflits en particulier, qui fonde la pertinence du système. Il s'est ainsi avéré relativement aisé de définir un ensemble d'États dont les liens étaient basés sur leurs positions antagonistes plus ou moins directes dans le conflit israélo-arabe. La forme du système fait alors apparaitre, d'une part, les pays de là confrontation, le cœur, le champ de bataille et, d'autre part, les pays qui organisent leurs relations de façon indirecte avec ce conflit nodal. Par ce biais, on peut distinguer d'un côté, la Syrie, l'Égypte, la Jordanie et le Liban, et de l'autre, l'Irak, l'Arabie Saoudite et les pays du Golfe. Cette perspective laisse de côté la Turquie et l'ensemble $\mathrm{du}$ Maghreb, ainsi que l'Iran (ce dernier pays pose toutefois problème du fait de son entrisme idéologique et paramilitaire dans le conflit, en particulier sur la scène sudlibanaise).

De ce point de vue, la définition du système régional trouve une certaine cohérence et des auteurs y ont décelé des «sous-systèmes" plus ou moins autonomes ${ }^{1}$. Pour notre propos, qui se limitera à l'économie et à certaines de ses traductions dans l'espace, les limites de ce système pourraient se définir à l'aune des différentiels d'intensité d'échanges, pour les pays limitrophes, entre l'intérieur et l'extérieur du système. Les échanges commerciaux comme les mouvements de capitaux peuvent en effet être 
discriminants pour en identifier des limites qui restent toutefois arbitraires. Cet exercice de délimitation géographique n'est en effet pertinent que dans la mesure où l'on s'attache à limiter l'investigation à la structure des réseaux et à leur fonctionnement en écartant d'emblée les relations internationales, l'histoire et la sociologie. Le système en cause est alors d'ordre économique et spatial et il ne préjuge pas des échanges multiples qui peuvent fonder la délimitation d'un ensemble plus vaste (monde arabe, monde islamique, etc.). Toutefois, dans la perspective des conséquences économiques d'une transformation géopolitique, il paraît cohérent, plutôt que de définir à tout prix une enveloppe géographique basée sur les échanges économiques, de fonder les limites du système en cause sur son assise politique, c'est-à-dire d'examiner les mutations qui ont lieu dans un espace de confrontation en cours de « conversion». Ainsi peut-on, dans l'optique d'une focalisation sur l'insertion israélo-palestinienne, concentrer l'observation sur les parties touchées, connectées à cette ancienne polarité, c'est-à-dire conserver la délimitation proposée par rapport au conflit israélo-arabe.

7 Il faut en premier lieu remarquer que la cohésion de ce système régional en termes économiques apparaît moins clairement que dans son cadre géopolitique, et les réseaux semblent peu densifiés. En ce qui concerne les seuls échanges commerciaux, un des indices permettant de mesurer le degré d'interrelations économiques, la cohérence est bien moindre. Pour reprendre la terminologie de Roger $B^{2}{ }^{2}$, on se trouve en présence d'un " espace mal intégré », « un espace dont les liens fonctionnent mal, dont les parties ont plus de relations avec l'extérieur qu'entre elles ». Le commerce extérieur des pays concernés est en effet largement polarisé, à des degrés divers, vers l'extérieur de ce système. Dans le cas de l'Égypte, la polarisation centrifuge est particulièrement accentuée : les importations et les exportations sont largement dominées par les pays industriels. Le commerce régional (dans les limites que nous nous sommes fixées) n'a, par exemple, compté que pour à peine $2 \%$ des importations et $8,6 \%$ des exportations de ce pays en $1992^{3}$.

8 Mais les échanges de biens, en dépit de leur trace physique, ne fondent pas à eux seuls l'ensemble des articulations du système économique. Pour être complet, il faut également examiner les flux de capitaux de différentes natures et les migrations de travail induisant des rapatriements de fonds.

9 Au Moyen-Orient, le transport des hydrocarbures, les mouvements financiers et de main-d'œuvre révèlent l'existence, sinon d'un ensemble véritablement intégré, du moins d'une interdépendance. Ces flux, différents par nature, ne sont pas l'apanage d'un ensemble régional clos : les hydrocarbures sont acheminés bien au-delà des limites $d u$ Moyen-Orient, et les flux de main-d'œuvre en provenance des pays du Sud-Est asiatique bousculent une délimitation trop stricte. En dépit de cette extraversion, qui illustre l'insertion mondiale d'une partie de l'économie régionale, il est possible de dégager des éléments qui contribuent à l'édification d'un réseau proprement endogène.

10 L'interdépendance des États peut être matérialisée par les réseaux d'acheminement pétrolier. Bien qu'aucun territoire de production pétrolière ne soit enclavé et que les pays producteurs soient tous dotés de façades maritimes, l'emplacement des champs et l'histoire de la prospection ont créé des réseaux multinationaux d'exportation. Du fait du conflit israélo-arabe et des tensions interarabes, les flux terrestres à destination de la Méditerranée ne sont plus utilisés. La Tapline, qui acheminait le pétrole saoudien vers la côte libanaise à travers la Jordanie, a été fermée en 1983 après les destructions occasionnées par l'invasion israélienne, ainsi que pour des questions de rentabilité. 
L'Irak s'est vu refuser l'accès à son débouché méditerranéen après la fermeture de l'oléoduc par le régime syrien, dès le début du conflit irako-iranien de 1980-88. Une partie importante des flux actuels, les exportations pétrolières saoudiennes vers l'Europe, demeurent tributaires du passage par l'isthme de Suez, ceci sous deux formes : par transport maritime à travers le canal et par l'oléoduc Sumed. En volume, elles dépassent 1,4 million de barils/Jour, sur un total d'exportations du royaume d'environ $6,5 \mathrm{mb} / \mathrm{J}: 570.000 \mathrm{~b} / \mathrm{j}$ représentent $41 \%$ du trafic pétrolier, nord-sud du canal de Suez ${ }^{4}$ et $840.000 \mathrm{~b} / \mathrm{j}$ empruntent le Sumed - ce qui totalise $62 \%$ du volume transporté par la canalisation ${ }^{5}$. Calculés en revenus pétroliers, ces volumes dépendants correspondent pour le royaume à un revenu compris entre 7 et 8 milliards de dollars par an pour un prix du baril compris entre 14 et 16 dollars. Autre cas significatif; l'Irak, qui ne bénéficie que de quelques kilomètres de débouché maritime sur le Golfe, dispose de trois moyens d'évacuation, tous tributaires d'une traversée extraterritoriale: l'oléoduc syrien (Kirkouk-Banyas), fermé; les oléoducs turcs et l'oléoduc qui traverse l'Arabie Saoudite jusqu'au terminal de Yanbu, sur la mer Rouge.

11 Les mouvements de capitaux ne sont pas non plus circonscrits au système régional, et l'on sait la part importante des capitaux arabes privés qui sont investis sur les places européennes, américaines ou asiatiques ${ }^{6}$. Mais les financements de projets, les soutiens aux balances des paiements, les transferts sans contrepartie publics ou privés sont des flux qui expriment une forme de complémentarité comptabilisée en balance de paiements courants d'un grand nombre de pays de la région. La redistribution de ce qu'on a appelé la rente pétrolière est le mécanisme financier le plus évident de ces interconnexions. Les surplus des balances commerciales des pays du Golfe sont en effet grevés par les postes des transferts: d'une part, les rapatriements de fonds des travailleurs émigrés d'Égypte, de Jordanie et du Liban; d'autre part, des transferts publics sans contrepartie, c'est-à-dire les aides fournies à ces mêmes pays par les monarchies du Golfe (voir tableau page suivante).

12 Les dernières évolutions constatées depuis la fin de la guerre du Golfe, à savoir, d'une part, le retour de fonds privés, après arbitrage entre les opportunités locales et les rendements obtenus sur des placements étrangers en dehors du Moyen-Orient et, d'autre part, le développement de places boursières régionales, confortent d'une certaine manière la densification du maillage. Les investissements des particuliers des pays du CCG contribuent certes, de façon encore marginale par rapport à l'encours des fonds disponibles, à conforter les connexions régionales, en apparaissant en mouvements de capitaux dans la balance de base des pays d'accueil. C'est le cas des investissements saoudiens en Égypte ou encore des rapatriements des Libanais du Golfe dans l'opération de souscription au capital de la société libanaise Solidere. D'après une étude de la Chambre de commerce de Riyad, les investissements saoudiens en Égypte atteignaient un montant de 3 milliards de livres égyptiennes en 1992, répartis dans 273 projets, ce qui représente $41 \%$ du total des investissements arabes dans le pays ${ }^{7}$. D'autre part, les expatriés libanais dans les pays du CCG ont souscrit à hauteur de 500 millions de dollars au capital de Solidere, sur un total de plus d'un milliard de dollars.

13 Enfin, les migrations de main-d'œuvre dans l'ensemble régional, polarisées sur les bassins d'emplois des pays rentiers de la péninsule, de la Libye pour l'Égypte, mais aussi de l'Irak avant 1990, déterminent une interdépendance forte. Les flux induits en retour, composés de rapatriements d'épargne, renforcent une dynamique d'échanges sujette, il est vrai, à des vicissitudes qui n'ont parfois rien d'économique. Depuis le milieu des 
années 80 et le contre-choc pétrolier, les pays du Golfe ont mené des politiques de diversification dans le recrutement de la main-d'œuvre immigrée, mettant fin à une domination de la force de travail originaire de la région. Les considérations économiques (coût de la main-d'œuvre) se sont mêlées en fait à des préoccupations politiques pour diminuer, dans la mesure du possible, le nombre des travailleurs en provenance des pays arabes au profit de ceux des pays du sous-continent indien et du reste de l'Asie. Les exemples les plus récents de cette sensibilité politique de l'immigration en sont l'expulsion indirecte d'Arabie Saoudite d'environ 700000 Yéménites après l'invasion du Koweït par l'Irak, et le retour du Koweït de près de 250000 Jordano-Palestiniens après la libération de ce pays, en 1991 et 1992. Dans le cas de l'Égypte, l'essor de l'exportation de main-d'œuvre date du milieu des années 70. Le nombre de travailleurs égyptiens à l'étranger était estimé à 390000 en $1975^{\circ}$ et aurait atteint, à l'apogée du boom des économies pétrolières de la région, près de $2000000^{9}$. En 1993, les Égyptiens seraient près de 1,5 million en Arabie Saoudite ${ }^{10}$ et représentent le premier contingent de travailleurs expatriés au Koweït, soit $150.000^{11}$.

Ainsi donc, l'espace économique régional, très imparfaitement intégré dans le domaine commercial, presque à la marge pourrait-on dire, n'en présente pas moins des caractéristiques propres à un système, dans la mesure où des flux s'organisent en quelque sorte en complémentarité, en rétroaction. Sans doute est-ce un système encore en émergence, en formation, dans une phase de densification et de ramification.

Les flux et la place de l'Égypte dans le système

15 L'Égypte n'est pas le cœur (y en a-t-il un, d'ailleurs ?) du système régional défini par des réseaux économiques. Toutefois, par sa position géographique et par sa démographie, elle est un élément central de dynamiques spatiales liées à des flux économiques.

16 1) Le facteur démographique est un des éléments dés des dynamiques. Ce n'est pas tant le poids de l'Égypte (bientôt 60 millions d'individus) que les mouvements induits par la pression démographique interne et l'appel d'air que constitue le relatif souspeuplement des pays pétroliers, qui font émerger les flux. Deux directions de nature différente peuvent être distinguées. L'une concerne des mouvements de main-d'œuvre, agricole et éducative ${ }^{12}$, et l'autre, en rétroaction, des mouvements de capitaux matérialisés par les transferts privés sans contrepartie. Pour des motifs différents, les chiffres sont difficiles à établir. L'existence de multiples taux de change en Égypte jusqu'en 1991 avait pour effet de diminuer considérablement, dans les statistiques officielles, les entrées réelles des rapatriements de fonds des travailleurs émigrés, qui s'effectuaient en dehors de toute régulation et dans des circuits parallèles plus rémunérateurs. Depuis deux ans, les chiffres enregistrés en balance des paiements courants font apparaître des volumes très élevés. Le rapport annuel de la Banque centrale d'Égypte indique, pour l'année fiscale 1991/92, des remises de fonds de 5,5 milliards de dollars, ce qui représente plus de trois fois les recettes d'exportations pétrolières ou 2,8 fois le montant perçu par l'organisme gestionnaire du canal de Suez. Par le volume financier de cette redistribution régionale de la rente, par le rôle qu'elle joue dans l'économie égyptienne mais aussi dans de nombreux pays hôtes, on peut considérer ces mouvements migratoires comme un fort élément structurant du système régional.

2) Le contrôle, par l'Égypte, de l'isthme de Suez, une connexion géographique stratégique, lui confère un rôle considérable dans les échanges d'hydrocarbures et une 
place de choix dans le système pétrolier régional. L'isthme est en effet le point de passage le plus rapide pour acheminer les hydrocarbures de l'Arabie Saoudite vers l'Europe (une quinzaine de jours en moins par rapport à la voie maritime du Cap). Son importance a été renforcée par la construction de la Petroline qui traverse d'est en ouest le royaume saoudien, dont la capacité s'élève à 4,7 millions de barils par jour au terminal de Yanbu. Par cette particularité, une position stratégique dans le réseau énergétique régional, l'Égypte est également fortement intégrée dans le maillage des flux.

18 3) L'intégration de l'Égypte dans l'économie de la rente pétrolière régionale ne se traduit pas uniquement par des mouvements de fonds de travailleurs émigrés. L'Égypte dispose aussi, selon les termes de Muhammad Sid Ahmad, d'une rente géopolitique ${ }^{13}$ qui lui donne accès à des aides et des financements des États-Unis et de l'Europe, mais aussi de partenaires arabes, du Golfe principalement. Pays de la "ligne de front", l'Égypte a pu bénéficier de subsides pendant sa confrontation avec Israël, sous la forme, pour la majeure partie des fonds, d'aide à la balance des paiements. Entre la guerre d'Octobre et Camp David, l'aide arabe annuelle fut en moyenne de 1,6 milliard de dollars, et elle représentait $22 \%$ du PNB en 1975 . Après la réintégration de l'Égypte dans la communauté arabe, le dernier geste de cette nature a été l'annulation, à l'automne 1990, de la dette extérieure égyptienne vis-à-vis des pays du Conseil de coopération du Golfe après son engagement dans la coalition qui a libéré le Koweït : un cadeau de 6 milliards de dollars. Aujourd'hui, aucune confrontation ne donne plus lieu à ce type de versements, et les difficultés budgétaires et financières, certes relatives, des pays du Golfe les ont incités à cibler davantage leur aide. Ce sont donc les financements de projets qui matérialisent désormais, au niveau étatique, ces flux financiers en direction de l'Égypte.

19 4) Autre aspect, autre origine des flux : le secteur touristique. Pour les populations des pays du Golfe, l'Égypte demeure un espace, un lieu de détente attractif. Leurs ressortissants, surtout saoudiens, représentent un des premiers contingents touristiques à destination de ce pays ${ }^{14}$. Sur les neuf premiers mois de 1993, le nombre d'arrivées s'établissait à 158.000 pour l'Arabie Saoudite, derrière la Libye; mais en nombre de nuitées, les Saoudiens étaient de loin les premiers de la liste, avec 1.300.000 nuitées enregistrées ${ }^{15}$. Les Palestiniens figuraient au troisième rang en nombre d'arrivées (75.000) et au quatrième en termes de durée de séjour (402.000 nuitées). Face à la crise que traverse l'Égypte depuis 1992, les autorités égyptiennes ont pris récemment la décision d'intensifier leur campagne de promotion à l'étranger, en y consacrant un budget sans précédent de 42 millions de dollars. La clientèle des pays arabes -et du Golfe en particulier - est une des cibles de cette initiative: elle a représenté en effet, pour l'année 1991/92, plus de 40\% du nombre des touristes et 43\% des nuitées ${ }^{16}$.

$\mathrm{Si}$, hormis les aspects politiques et géopolitiques que nous n'évoquerons pas ici, l'Égypte n'occupe pas véritablement une place centrale dans ce système économique moyen-oriental, elle n'y joue pas non plus un rôle marginal dans la dynamique des flux multiples brièvement décrits ici. C'est un élément structurant qui entre en interactions fortes avec un milieu par ailleurs dépourvu de véritable corps centripète. Nous allons tenter d'évaluer maintenant ce que pourraient être les conséquences d'une modification notable des structures et l'apport de connexions supplémentaires dans ce système sur l'élément égyptien. 
L'insertion israélo-palestinienne

21 Deux éléments à caractère étatique ou quasi-étatique doivent faire leur apparition dans le système : l'État d'Israël, dont les relations politiques seraient alors normalisées avec son environnement arabe, et l'État de Palestine, dont le processus de création, malgré les vicissitudes, est enclenché.

Pour mesurer en premier lieu l'impact de l'insertion de l'économie israélienne dans l'espace proche-oriental, ou plus exactement dans l'ensemble des connexions géoéconomiques, il faut au préalable se situer à deux niveaux d'échelles, en en identifiant les caractéristiques principales :

- C'est avant tout une économie industrielle et tertiaire fortement liée à l'Europe et aux ÉtatsUnis, qui dispose de connexions extrarégionales très fortes. Le taux de pénétration de l'économie israélienne, mesuré par le rapport entre les importations de biens et services sur le produit national brut, est d'environ 35\%. Les produits industriels (sans les diamants, qui représentent le quart des exportations) comptent pour $67 \%$ des exportations totales du pays. D'autre part, les aides en provenance des pays développés (États-Unis, Allemagne surtout) sont parmi les plus élevées pour un pays de cette catégorie de revenu, et se montent ces dernières années à plus de 5 milliards de dollars par an, soit l'équivalent d'un tiers du montant des exportations de biens et services et plus de 10\% du PNB. La polarisation extrarégionale se fait donc essentiellement vers les États-Unis et l'Europe, avec laquelle Israël réalise plus des trois quarts de son commerce extérieur et $80 \%$ de ses importations. En cela, son économie offre une similitude avec l'ensemble des pays de la région, qu'ils soient producteurs de pétrole ou non, dans l'extraversion de leur commerce extérieur vis-à-vis de la zone. Au cours de la décennie 80 en effet, le commerce extérieur (importationexportation) des pays de la Ligue arabe hors pays membres n'a dépassé les $20 \%$ que pour trois d'entre eux : le Soudan, la Jordanie et le Liban ${ }^{17}$.

- C'est aussi une façade méditerranéenne à la recherche d'un hinterland. Les ports de la côte palestinienne ont joué, avant la création de l'État d'Israël, un rôle de rupture de charge avec les flux en provenance de la Syrie et du désert nord-arabique, et les premiers oléoducs transrégionaux d'évacuation vers la Méditerranée, la Tapline et l'oléoduc nord-irakien aboutissaient au terminal de Haïfa. L'intégration économique israélienne devra s'opérer par la quête de cet hinterland.

23 C'est, dès lors, à une véritable révolution de son tropisme économique que l'économie israélienne doit se livrer pour réaliser son intégration. Autant dire que ce mouvement sera lent et partiel, car la structure est pesante et la concurrence proche. En effet, la constitution d'une nouvelle porte d'entrée pour les flux Europe/Moyen-Orient est également l'objectif et la vocation de la place de Beyrouth, en cours de réhabilitation, qui tente de faire renaître son savoir-faire dans ce domaine.

Quels pourraient être en second lieu les axes de développement de l'économie palestinienne? Avant toute tentative sur ce point, il faut d'abord constater que la prospective reste particulièrement périlleuse, au regard de la faible fiabilité des chiffres, d'une part, et de l'incertitude totale concernant la situation politique, d'autre part. Il est en effet prématuré de former des stratégies de développement sans connaître exactement les limites du territoire, les cadres institutionnels, la nature des relations avec l'extérieur. Toutefois, il est possible d'imaginer en quelques mots, et dans une vision d'assez long terme, les possibilités de développement de l'élément palestinien dans la structure globale du système et dans ses articulations. 
La structure de l'élément palestinien sera bipolaire, territorialement et économiquement : deux entités spatiales, une enclave et un territoire riverain, forment le couple palestinien. Il est impératif de rappeler d'abord la dépendance très forte vis-àvis d'Israël : plus de $80 \%$ du commerce extérieur de la CisJordanie et de Gaza sont réalisés avec ce pays et, avant les mesures d'interdiction d'entrée prises en 1993 par les autorités israéliennes, $40 \%$ des travailleurs de la bande de Gaza exerçaient en Israël, et $30 \%$ pour la CisJordanie. La première nécessité, politique autant qu'économique, sera donc d'établir des connexions, de maîtriser des flux directs vers l'extérieur, vers les pays arabes bien sûr, mais aussi vers les pays occidentaux. Mais l'ampleur de la reconnexion ne devrait pas être telle que ces attaches soient rompues, et il est probable que c'est malgré tout en développant une industrie de sous-traitance performante, orientée d'ailleurs aussi bien vers le marché israélien que vers le reste de la région, que les Palestiniens ont une carte à jouer. Celle-ci pourrait se coupler à un pôle méditerranéen actif, autour d'une zone portuaire dont les spécialisations restent à définir.

Une des perspectives de développement de Gaza se fonde sur la création d'une enclave portuaire de réexportation et d'importation pour la région : un «Singapour » arabe ? ${ }^{18}$. Les conditions de l'émergence d'un tel centre sur ce modèle asiatique sont la présence d'un hinterland densément peuplé, une position stratégique sur des routes commerciales internationales et l'existence d'un pôle bancaire et de services aux connexions internationales très fortes. La combinaison de ces trois prémisses n'est pour l'instant pas réalisée par l'environnement palestinien. Toutefois hinterland démographique existe bien, en particulier au sud de Gaza, où l'Égypte offre un marché potentiel avec ses 58 millions d'habitants. Mais l'instauration d'un pôle financier et une position commercialement stratégique devront compter avec les sites déjà nombreux sur ce pourtour méditerranéen, interface entre l'Europe du Sud et le Moyen-Orient.

Quant à la Cisjordanie, c'est un ensemble enclavé entre Israël et la Jordanie, un espace à faible capacité d'absorption démographique et à capital limité. Son économie pourrait y trouver des niches sur un marché régional compétitif et sur des marchés extrarégionaux non moins disputés. C'est probablement la combinaison, la complémentarité du pôle offshore ou méditerranéen, d'un côté, et de l'ensemble continental, de l'autre, qui permettra de réaliser un développement complémentaire et cohérent dans cet ensemble économique palestinien encore au stade embryonnaire.

Les nouveaux flux et leurs conséquences sur l'Égypte

On a pu noter par ailleurs le faible impact de la paix égypto-israélienne dans le domaine économique. Aussi faut-il être prudent sur celui qui émanerait d'une paix arabeisraélienne plus globale. En effet, l'ouverture des frontières et la disparition des barrières psychologiques et politiques n'impliquent pas forcément le renforcement des structures de connexion, car des facteurs de polarisation pèsent lourdement sur la dynamique propre de chaque entité territoriale.

Pour qu'il y ait, de façon générale, un effet déstructurant sur le pôle égyptien, il faudrait que les nouveaux éléments intégrés dans le système, par le déséquilibre qu'ils amèneront immanquablement, soient susceptibles de capter à leur profit des flux qui partent ou qui entrent en Égypte. Au regard de l'analyse qui va suivre, cette hypothèse ne nous paraît pas fondée.

L'économie palestinienne, par ses structures et son fonctionnement, par son tropisme surtout, ne devrait pas nuire au développement des échanges commerciaux de l'Égypte 
et à ses productions locales, qui disposent d'un vaste marché en propre et dont le commerce extérieur est encore largement régi par des structures protectionnistes. Mais les contraintes de l'environnement international (les accords du GATT en particulier) ainsi que celles de la balance des paiements et du développement, amènent les autorités et les milieux d'affaires égyptiens à s'interroger sur les réformes nécessaires dans ce domaine. Dans la perspective d'une ouverture plus large de son économie, l'Égypte - en particulier son secteur industriel - sera soumise à des défis qui ne pourront être relevés que par une refonte des structures de production et d'exportation. La réponse à l'ouverture aux produits israéliens et palestiniens se pose là en termes de compétitivité sur le marché intérieur. Mais à bien des égards, et dans le secteur primaire surtout, les productions de ces trois économies sont similaires, et la confrontation devrait se faire dans une autre arène. Le problème de l'insertion des économies israélienne et palestinienne ne se pose alors sans doute pas tant en termes de pénétration et de concurrence sur le marché égyptien même, qu'en termes de captation des marchés d'exportation.

31 Dans des domaines concurrentiels à l'exportation (fruits et légumes, artisanat, textile), les coûts plus élevés de la main-d'œuvre palestinienne et, plus encore, israélienne, devraient jouer en faveur de l'Égypte. Toutefois, dans le cas du développement d'une industrie de haute technologie ${ }^{19}$, dont l'apparition est encore bien hypothétique en Palestine, la formation des cadres palestiniens et leur internationalisation, conférerait à ce secteur des avantages comparatifs non négligeables mais devant se positionner, là comme dans d'autres domaines, face à une concurrence israélienne forte et structurée.

En ce qui concerne la concurrence sur les marchés de main-d'œuvre dans les pays pétroliers, la situation actuelle et passée montre que les Égyptiens et les Palestiniens n'occupent pas les mêmes emplois. Dans l'hypothèse de modifications de l'écosystème démographique local, pour reprendre les termes d'une analyse développée par Philippe Fargues $^{20}$, les changements opérés dans le sous-système migratoire du fait d'un retour massif de Palestiniens de l'extérieur pourrait toutefois affecter les Égyptiens, dans la mesure où le départ de cadres palestiniens vers la Palestine formerait un appel d'air en direction des cadres égyptiens. Cet impact est bien sûr très difficile à évaluer.

En ce qui concerne les flux de capitaux publics et privés, il faut raisonner en termes de substitution d'axes de redistribution et de reconnexions liées à d'éventuels changements de priorité stratégiques et économiques. L'intégration de l'économie israélienne et de l'économie palestinienne va-t-elle modifier le comportement des acteurs internes et externes au système régional dans le domaine financier? En clair, les flux d'aides vont-ils être réaménagés et, si oui, cela se fera-t-il au détriment ou au bénéfice de l'Égypte? Celle-ci, sous la direction du président Moubarak, sait se montrer indispensable à l'équilibre géopolitique de la région, et la stratégie américaine de contrôle des sources d'approvisionnement énergétique, en particulier saoudien, ne devrait pas conduire à une remise en cause de l'appui qu'elle apporte à l'un des piliers dans la région. L'aide américaine n'est pas liée à la situation de belligérance mais à la nécessité de conforter cet allié, ses capacités de résister à la déstabilisation interne et d'épauler le déploiement des forces extérieures sur des terrains conflictuels, comme t'a montré la guerre du Golfe. Les aides occidentales qui accompagnent l'ajustement économique entrepris par l'Égypte depuis 1991 ne devraient pas quitter le navire. L'Égypte, pôle de stabilité politique, joue de cette rente géopolitique dans une région que les analystes des cabinets ministériels occidentaux estiment menacée par la 
montée des islamistes extrémistes. Depuis 1975 , l'économie égyptienne a reçu 12,8 milliards de dollars de dons de la part des États-Unis. Le montant de l'aide en 1994 est de 2,1 milliards de dollars, dont 1,3 d'aide militaire.

Du côté arabe, l'aide n'est plus liée à la situation conflictuelle avec Israël comme ce fut le cas jusqu'en 1978. Réintégrée sur la scène politique arabe à la fin des années 80, l'Égypte a pu bénéficier d'une aide importante, mais plus ciblée, essentiellement constituée de financements de projets. Tous ces bailleurs de fonds vont être sollicités par la construction de l'économie palestinienne et les engagements se sont multipliés, bien que leur montant - représentant pour l'instant quelque 2 milliards de dollars ${ }^{21}-$ soit bien en-deçà des espérances des principaux intéressés. Il est douteux que des arbitrages publics soient réalisés au détriment de l'Égypte, pays aux besoins immenses et à l'importance géopolitique capitale.

La lisibilité des arbitrages qui seront effectués par les détenteurs de capitaux privés est très faible, la principale motivation étant ici le rendement financier des placements et des investissements. A cet égard, la politique monétaire suivie en Égypte même sera décisive. La stabilité de la livre depuis 1991 et les taux d'intérêts élevés ont joué en faveur des placements en Égypte, mais le contrecoup en est une surévaluation en termes réels par rapport aux principales devises. La conséquence bénéfique - le prix relativement bas des importations - se retourne contre l'Égypte dans le cadre d'une compétition régionale renforcée à l'exportation. La stratégie de positionnement de l'Égypte sur les marchés extérieurs pourrait donc avoir des incidences notables sur les flux de capitaux privés vers son système bancaire. L'arbitrage en faveur de la Palestine ou d'Israël reste incertain dans un proche avenir. Tant que la Palestine n'offrira pas de structures bancaires et juridiques ${ }^{22}$ pour attirer de tels fonds, il serait vain d'escompter de quelconques mouvements massifs et continus dans cette direction. Il faut également tenir compte de la volonté politique d'autres membres du système de fonctionner sur ce réseau - Dubaï dans le Golfe et Beyrouth sur la Méditerranée - ce qui ne facilitera pas l'émergence d'un tel pôle.

Le survol des nouvelles articulations des flux de marchandises, de capitaux et de maind'œuvre montre que l'intégration palestino-israélienne n'apportera pas de changements majeurs dans les connexions économiques de l'Égypte. S'il y a des modifications induites par de nouveaux flux, elles seront davantage de type endogène, c'est-à-dire qu'elles affecteront la façon dont les Égyptiens répondent, par des réformes de structure, à l'émergence d'un marché régional plus ouvert et plus intégré, dans le sens d'une plus grande introversion. S'il y a toutefois un secteur moins préservé, c'est celui des flux régionaux de produits pétroliers, car les ruptures de l'équilibre actuel du système pourraient affecter la structure du réseau égyptien et ses connexions régionales.

Sans entrer ici dans les détails, il faut cependant remarquer que l'impact de la réouverture des oléoducs Golfe-Méditerranée ${ }^{23}$, d'une part, et l'apparition d'un axe Yanbu-Rafah, d'autre part, représenteraient, en termes systémiques, des connexions supplémentaires plus que de substitution. Dans une perspective à moyen et long terme du marché pétrolier mondial, qui place le Moyen-Orient dans une position renforcée de monopolisation de la ressource sur le plan mondial, ces nouveaux synapses constitueraient plutôt un renforcement de la structure du réseau actuel ${ }^{24}$ et non pas une réorientation des flux à même de dénaturer les connexions établies par l'Égypte. 
Au contraire même, puisque, dans l'hypothèse d'une ligne débouchant sur Gaza, le rôle de ce pays s'en trouverait renforcé.

Du système au territoire

L'ensemble des reconnexions liées à l'insertion des économies et des territoires d'Israël et de Palestine dans la région ne devrait finalement pas remettre en cause une grande partie de la structure des flux qui irriguent l'économie égyptienne. Qu'elles aient ou non une empreinte dans l'espace économique, les nouvelles relations devraient n'affecter qu'à la marge le fonctionnement du maillage qui conforte la position et la situation stratégique de l'Égypte et assure encore, in fine, son développement économique. Mais pour mesurer pleinement l'impact de ces nouvelles dynamiques, il faut pouvoir changer d'échelle et de grille de lecture. En définitive, l'économie autant que l'espace sont ici en jeu, qu'il s'agisse du canal de Suez, des réseaux et des infrastructures de transport ou de la mobilité des agents économiques. A petite échelle graphique. dans un regard qui englobe l'ensemble du Moyen-Orient, l'Égypte préserve ses positions, et sa permanence - sinon sa pesanteur - lui confère une assurance contre les bouleversements auxquels pourrait être confrontée par exemple la Jordanie. Mais l'Égypte n'est pas pour autant exempte de défis. Car s'il existe une forte connexion potentielle, c'est bien celle qui se dégage de la contiguïté de deux espaces. Or le territoire égyptien côtoie, par-delà le Sinaï, à la fois Israël et la bande de Gaza. C'est un face-à-face désertique dont il s'agit, mais le Néguev comme le Sinaï sont des espaces pionniers et l'axe littoral qui court du nord de la Syrie au delta du Nil pourrait se renforcer sur l'espace intermédiaire situé entre Port Saïd et Gaza.

Les travaux d'Olivier Sanmartin ${ }^{25}$ ont montré comment le gouvernement égyptien a mis en œuvre des moyens de différentes natures après la rétrocession progressive du Sinaï par Israël pour réintégrer un espace jusqu'alors marginal (mais aussi un glacis) sur le territoire égyptien. L'ouverture plus systématique de la frontière commune avec le Néguev, mais surtout l'émergence d'un pôle de développement à Gaza. pourraient modifier de façon sensible la polarisation de cet espace et une partie plus large du territoire égyptien. Cette connexion en devenir avec le territoire palestinien de Gaza est en tous cas l'objet de commentaires et de visions futuristes, de l'édification de voies ferrées ${ }^{26}$ à l'apparition de flux touristiques pendulaires ${ }^{27}$ en passant par la création d'aéroports ${ }^{28}$.

Le déséquilibre provoqué par cette connexion peut donner lieu selon nous à deux types de réactions du « système » égyptien : soit l'intégration proche-orientale provoque une connexion très forte, densifiée en termes de structures, avec l'espace palestinien et israélien, dans une dynamique de rétroaction positive qui accentuerait le détachement relatif du Sinaï vers le pôle extérieur; soit la rétroaction négative s'opère par la reconnexion symétrique inverse du territoire péninsulaire vers le corps égyptien, dans un mouvement qui ferait du Sinaï un véritable espace relais entre l'Égypte et l'espace palestino-israélien. C'est un des défis majeurs auxquels seront confrontés les politiques et les planificateurs égyptiens. La réunion qui s'est tenue à la fin du mois de janvier $1993^{29}$ entre les gouverneurs du Sinaï et le gouvernement témoigne de préoccupations bien réelles. N'y était-il pas question de mettre le Sinaï en « salle de réanimation " pour faire face aux conséquences de l'accord Gaza-Jéricho?

41 Article tiré d'une intervention au colloque sur L'économie égyptienne et les perspectives de paix globale au Proche-Orient organisé par le Center for Economie and Financial Research and Studies de l'Université du Caire et le CEDEJ les 11 et 12/12/93. 


\section{ANNEXES}

Transferts publics et privés sans contrepartie dans la balance des paiements

Exemple de l'Arabie Saoudite, du Koweït, de l'Égypte et de la Jordanie

\begin{tabular}{|c|c|c|c|c|c|}
\hline en millions \$ & 1988 & 1989 & 1990 & 1991 & 1992 \\
\hline \multicolumn{6}{|l|}{ Arable Saoudite } \\
\hline Balance commerciale & 4.510 & 9.068 & 22.806 & 21.648 & 12.991 \\
\hline Transferts privés sans contrepartie & -6.510 & -8264 & -11.602 & -14.117 & -12.700 \\
\hline Transferts publics sans contrepartie & -2.499 & -2200 & -4.401 & -6.489 & -1.501 \\
\hline Balance des services & -12580 & -17.490 & -1.415 & -35.877 & -25220 \\
\hline Balance courante & -7.340 & -9.172 & -4.267 & -27.730 & -19.431 \\
\hline \multicolumn{6}{|l|}{ Koweït } \\
\hline Balance commerciale & 2.261 & 5.872 & 3.540 & -3226 & 549 \\
\hline Transferts privés sans contrepartie & -1.179 & -1283 & -770 & -503 & -870 \\
\hline Transferts publics sans contrepartie & -140 & 211 & -4.181 & -23.372 & -1.067 \\
\hline Balance des services & -3.053 & -2.774 & -2.035 & -3.958 & -2.064 \\
\hline Balance courante & 5.208 & 9.688 & 4.042 & -25.891 & 801 \\
\hline \multicolumn{6}{|l|}{ Égypte } \\
\hline Balance commerciale & -6.608 & -5.933 & -6.699 & -5.975 & -5.501 \\
\hline Transferts privés sans contrepartie & 3.770 & 3.293 & 4.284 & 4.054 & 6.104 \\
\hline Transferts publics sans contrepartie & 666 & 800 & 1.119 & 1.380 & 972 \\
\hline Balance des services & 1.326 & 1.129 & 2.497 & 3.722 & 3.101 \\
\hline Balance courante & -1.048 & -1.309 & 184 & 1.903 & 2.812 \\
\hline \multicolumn{6}{|l|}{ Jordanie } \\
\hline Balance commerciale & -1.411 & -773 & -1.237 & -1.173 & -1.780 \\
\hline
\end{tabular}




\begin{tabular}{|l|l|l|l|l|l|}
\hline Transferts privés sans contrepartie & 800 & 565 & 457 & 408 & 781 \\
\hline \hline Transferts publics sans contrepartie & 552 & 613 & 588 & 476 & 386 \\
\hline \hline Balance des services & 80 & 176 & 179 & 229 & 124 \\
\hline \hline Balance courante & -294 & 385 & -227 & -394 & -765 \\
\hline
\end{tabular}

Source : International Financial Statistics, mai 1994.

NB : Les chiffres des balances des paiements indiqués dans le tableau n'expriment pas uniquement les relations à l'intérieur du système moyen-oriental, puisqu'il s'agit de données sur l'ensemble du monde. Ainsi les importants transferts publics de l'année 1991 sont-ils le reflet des sommes allouées aux partenaires arabes mais surtout occidentaux de la guerre du Golfe. Ils mettent toutefois en relief les structures presque opposées des principales composantes des balances des paiements courants et, d'une certaine façon, leur complémentarité, notamment pour ce qui relève des transferts.

\section{NOTES}

1. Par exemple, Salame Gh. dans sa thèse sur le développement du rôle régional et international de l'Arabie Saoudite, Université de Paris 1, 1978, 2 vol., 594 p.

2. Brunet R. (éd.), Les mots de la géographie, Reclus, La Documentation française, Paris, $472 \mathrm{p}$.

3. D'après des calculs effectués à partir des données de Direction of Trade Statistics Yearbook, FMI, 1993. Voir également sur ce point : Fishers S., Rodrik D., Tuma E, (éd.), the Economies of Middle East Peace : Views from the Region, MIT. 1993, 370 p. : "the Economies of Peace : the Egyptian Case", par Heba Handoussa et Nema Shafik, pp. 19-54.

4. Pétrostratégies. 28/6/93.

5. Majallat al-batrûl, vol. 29, n012, décembre 1992, p. 45.

6. Le directeur du Fonds monétaire arabe estimait à 650 milliards de dollars les investissements arabes hors de la zone en décembre 1993.

7. Al-Hayat, 8/2/94.

8. Birks and Sinclair, mai 1979, USAID contract $n^{\circ}$ AIB/NE-C-1593.181 $p$.

9. Alibon R., the Red Sea Region, Londres, Croom Helm, 1985,143 p.

10. Al-Ahrâm, 17/1/94.

11. Al-Wasat, 31/1/94, p.19.

12. Selon des chiffres publiés par le Bureau égyptien de représentation de la maind'œuvre et de l'emploi à Ryad, le volume de la main-d'œuvre égyptienne en Arabie Saoudite serait de 900.000 individus.

13. « Nous sommes une économie de services géostratégiques (...). La paix avec Israël nous avait valu une aide considérabe de l'Occident. Maintenant, nous monnayons la guerre du Golfe. » Entretiens avec Le Figaro, 30 novembre 1990, cité dans Seguin J., "L'économie égyptienne après la guerre du Golfe », Maghreb-Machrek n 133, juilletseptembre 1991, p. 38.

14. Voir à ce sujet l'enquête d'Al-Hayat. 19/10/93. 
15. Voir l'enquête de Farid Al-Qâdi dans Le Progrès Égyptien, 18/11/93.

16. Central Bank of Egypt, Economic Review, vol. XXXII, n 4,1991/92, p. 191.

17. Fargues Ph. et Boustani R., «Place des partenaires des pays arabes dans leur commerce international », Atlas du Monde arabe, Bordas, 1990, p. 101.

18. 'Abd Allah R., «Ayya «Singapour falastîniyya» nurîdu ? ", al-Hayat, 23/10/93.

19. Voir à ce sujet les projets de " vallée technologique » du gouverneur d'Ismâ'iliyya, dans Al-Ahram Weekly, 11-17/11/93.

20. Communication présentée au Caire le 9 novembre 1993 dans le cadre du cycle de conférences du CEDEJ.

21. Middle East Economic Digest, 17/12/93, pp. 2-3.

22. II s'agirait de faire de Gaza un « des centres bancaires et financiers les plus importants au Moyen-Orient et d'attirer les investissements et les capitaux des pays arabes ", comme le souhaite un responsable palestinien interrogé par Reuter dans $\mathrm{Al}$ Hayat, 10/11/93.

23. «La paix au Moyen-Orient fait peser de lourdes menaces sur le Canal de Suez et le Sumed », Le pétrole et le gaz arabes, 1er octobre 1993, p, 20 et Al-Hayat, 24/10/93.

24. Cf. « Nouvelles options énergétiques » et « Israël prépare son intégration énergétique au Moyen-Orient », Pétrostratégies n³60,18/10/93, pp. 1 et 6-7; Blin L, « Les hydrocarbures du Golfe et les perspectives énergético-industrielles d'un Proche-Orient en paix ", in Blin L., Fargues P. (éd.), L'économie de la paix au Proche-Orient, tome I, Stratégies, Paris, Maisonneuve et Larose, 1995.

25. Sanmartin O., Processus et modalités d'intégration d'un espace périphérique : le Sinaï, mémoire de DEA, Université de Tours, 1993.

26. Middle East Monitor, novembre 1993 : selon la radio israélienne, l'ambassadeur égyptien en Israël, Muhammad Basiûnî a indiqué au ministre israélien des Transports, en octobre, que les autorités égyptiennes étaient intéressées par la réouverture du chemin de fer Le Caire-Tel Aviv.

27. Al-Ahram Weekly, 21-27/10/93 : "Political Moves in Sinai”. Les récents changements qui traversent le Moyen-Orient vont, selon certains experts, avoir un impact décisif sur le développement touristique, du fait que le nord du Sinaï borde la bande de Gaza. Un effet immédiat a été la hausse vertigineuse des prix des terrains à Rafah, qui se sont mutipliés par quatre après la signature de l'accord Gaza-Jéricho. On prévoit également que le nombre de personnes franchissant la frontière à Rafah - le seul débouché de Gaza sur le monde arabe - passera de 250000 actuellement à 1 million.

28. Le Progrès Égyptien, 2/11/93; «L'OLP et l'Égypte ont l'intention de construire un aéroport commun dans la bande de Gaza et le désert du Sinaï, a-t-on déclaré hier de sources proches de la centrale palestinienne. L'aérogare se trouvera à Rafah et les pistes dans le Sinaï, ajoute-t-on de même source en précisant que les deux parties tentent d'obtenir une aide de la Banque mondiale. »

29. Al-Ahrâm, 20/1/94. 
INDEX

Mots-clés : économie, Moyen-Orient, paix

\section{AUTEUR}

JACQUES SEGUIN

CEDEJ 